\title{
Ratiometric pH Measurements Using LysoSensor DND-192
}

\author{
Jung Sook Kang* and Yordan Kostov ${ }^{\dagger}$ \\ Department of Oral Biochemistry and Molecular Biology, College of Dentistry and Research Institute for Oral Biotechnology, \\ Pusan National University, Pusan 602-739, Korea \\ 'Department of Chemical and Biochemical Engineering, University of Maryland Baltimore County, \\ 1000 Hilltop Circle, Baltimore, MD 21225 USA
}

Received 12 March 2002, Accepted 23 April 2002

\begin{abstract}
A method for the ratiometric pH sensing using LysoSensor DND-192 is presented in this paper. It works in the physiological $\mathrm{pH}$ range. It is based on the use of two fluorophores which differ significantly in their lifetimes. As the discrimination of their emissions is performed through two different frequencies, this method can allow significant overlap of the emission spectra. A simple long-pass filter, or a combination of long- and short-pass filters, was used instead of narrow-bandpass devices. Importantly, the measurements were carried out under strong ambient light. The method could be used in a wide variety of applications, such as intracellular measurements, microscopy, bioprocess monitoring, etc.
\end{abstract}

Keywords: Ratiometric pH sensing, LysoSensors, Dualfrequency modulation, Ambient light exclusion

\section{Introduction}

Fluorescence sensing techniques are widely used for highsensitivity detection in cell biology (Bae and Lee, 2001; Lee and Lim, 2001; Uh et al., 2001). Recently, fluorescence measurements of $\mathrm{pH}$ have gained a lot of attention since they allow intracellular measurements. But, the only fluorescence parameter for many $\mathrm{pH}$-sensitive dyes that change is their emission intensity. Fluorescence intensity measurements are prone to errors because they depend on a number of factors. These include the intensity of excitation light, length of optical paths, concentrations of fluorescent dyes, primary and secondary inner filter effects, (self-) quenching, etc. (Lakowicz, 1999). In order to avoid extensive and laborious calibration, self-referencing fluorescence methods are used. One example is the lifetime measurement. It is free from many of the problems that were previously mentioned,

*To whom correspondence should be addressed.

Tel: 82-51-240-7820; Fax: 82-51-241-1226

E-mail: jsokang@pusan.ac.kr however, most of the available fluorescent $\mathrm{pH}$ indicators have short lifetimes $\left(10^{-8} \sim 10^{-11} \mathrm{~s}\right)$. The equipment for lifetime measurements on this time scale is complex and sophisticated (Lakowicz et al., 1986; Laczko et al., 1990). Furthermore, the fluorescent dyes that have vastly different lifetimes of the protonated and unprotonated forms are needed. If they differ only slightly or one of the forms is not fluorescent, then the lifetime measurements of $\mathrm{pH}$ become impractical or even impossible. Another well-known possible approach is to use ratiometric probes (Nedergaard et al., 1990; Whitaker et al., 1991; Slavik et al., 1999; Zignani et al., 2000). In this case, narrow bandwidth devices (filters or monochromators) are required for the separation of the excitation or emission bands. Otherwise, the overlap of the spectra strongly deteriorates the sensitivity of the measurement. A narrow bandwidth results in low-light intensities, which imposes the use of highly sensitive photodetectors (photomultipliers or CCD-based detectors).

Modulation sensing is one solution for avoiding intensityrelated problems when using intensity based dyes (Lakowicz et al., 1998; Abugo et al., 2000). The used fluorophores greatly differ in lifetimes, so their spectra can significantly overlap. Because the measurements of modulation are generic, it is possible to use them in a variety of applications. However, this method requires the exclusion of ambient light or at least to account for it. In real-life applications, this is not always possible, or else it significantly complicates the measurement procedures. This makes things difficult or prevents its application in a number of fields, such as bioprocess monitoring, microscopy, etc.

Recently, Molecular Probes (Eugene, USA) introduced a new family of cell permeable $\mathrm{pH}$ sensitive dyes LysoSensors, and their lifetime responses were characterized (Lin et al., 2001). Although the cells readily take up the dyes, it is difficult to use them. This is because none of the family exhibits ratiometric properties, except LysoSensor DND-160 that is a dual-excitation and dual-emission probe. In this paper, we present the use of intensity-based LysoSensor DND-192 for the ratiometric $\mathrm{pH}$ measurements. We used a 
new approach for the ratiometric $\mathrm{pH}$ sensing that is based on the use of two fluorophores with significantly different lifetimes. An in-house-built device was used for method implementation. This method requires no narrow-bandpass devices (monochromators or interference filters), but it relies on simple long-pass filtering. Furthermore, the method is intrinsically insensitive to ambient light.

Theory The theory for ratiometric measurements that uses dual-frequency lifetime discrimination has been described as follows (Kostov et al., 2000, 2001). Let a fluorescent sample emit two emissions with steady-state intensities $A_{1}$ and $A_{2}$ and lifetimes $\tau_{1}$ and $\tau_{2}$ and $\tau_{1}=1000 \tau_{2}$, respectively. The ratio $A_{1} /$ $A_{2}$ is to be determined.

If the sample is excited by modulated light, the $\mathrm{AC}$ amplitude $A$ of the emission will be dependent on the excitation frequency, the lifetimes, and the steady-state intensities by the equation

$$
A=\frac{A_{1}}{\sqrt{1+\omega^{2} \tau_{1}^{2}}}+\frac{A_{2}}{\sqrt{1+\omega^{2} \tau_{2}^{2}}}
$$

Suppose that $\omega_{1}=0.1 / \tau_{1}$. Then

$$
A_{\omega_{1}}=0.995 \cdot A_{1}+A_{2} \approx A_{1}+A_{2}
$$

Therefore, at very low frequencies the amplitude of the total emission equals the sum of both partial emissions. Now, suppose that $\omega_{2}=100 / \tau_{2}$. Then

$$
A_{\omega_{2}}=0.0099 \cdot A_{1}+0.995 \cdot A_{2} \approx A_{2}
$$

In other words, there is a range of frequencies at which the amplitude of the long-lifetime emission is practically zero. When the sample is excited at these frequencies, the AC amplitude of the emission is almost exclusively from the short-lived fluorophore.

As a result, the ratio $\left(A_{1} / A_{2}\right)$ of steady-state intensities of the two emissions (regardless of the degree of overlap between the spectra) can be determined by the equation:

$$
\frac{A_{1}}{A_{2}}=\frac{A_{\omega_{1}}}{A_{\omega_{2}}}-1
$$

\section{Materials and Methods}

Materials LysoSensor DND-192 was purchased from Molecular Probes (Eugene, USA). [Ru(bpy) $\left.{ }_{3}\right] \mathrm{Cl}_{2}$ (bpy $=2,2$ '-bipyridine) and all of the other chemicals that were used for preparing the $\mathrm{pH}$ buffers (such as citrate, phosphate, Tris, and bicarbonate) were obtained from Sigma-Aldrich (St. Louis, USA). The buffer concentrations were kept at $50 \mathrm{mM}$ in the presence of $0.1 \mathrm{mM} \mathrm{KCl}$ in order to maintain a constant ionic strength. A stock solution of LysoSensor DND-192 was first prepared in distilled water at a concentration of $0.1 \mathrm{mM}$, then diluted with $\mathrm{pH}$ buffers to a final concentration of $20 \mu \mathrm{M}$. The reference film consisted of $1 \%(\mathrm{w} / \mathrm{w})\left[\mathrm{Ru}(\mathrm{bpy})_{3}\right] \mathrm{Cl}_{2}$ that was physically entrapped in cellulose acetate. In order to prevent leaching $\left[\mathrm{Ru}(\mathrm{bpy})_{3}\right]^{2+}$ from the film, it was protected from contact with water by a thin layer $(0.2 \mathrm{~mm})$ of RTV108 silicone rubber.

Steady-state and time-resolved fluorescence measurements The fluorescence excitation and emission spectra were measured using a SLM Model 8000 spectrofluorometer (Spectronic Instruments Inc., Rochester, USA). The excitation wavelength was $370 \mathrm{~nm}$, and the emission wavelength was set at $425 \mathrm{~nm}$. Frequency-domain lifetime measurements were performed with an ISS Koala instrument (ISS, Champaign, USA) using a UV lightemitting diode (LED) NSHU590E (Nichia America, Mountville, USA) as the excitation source. A $370 \pm 10 \mathrm{~nm}$ interference filter and a $450 \pm 50 \mathrm{~nm}$ interference filter were used for isolating the excitation and emission, respectively. As a lifetime standard, $p$ $\operatorname{bis}(O$-methylstyryl)benzene in cyclohexane $(\tau=1.68 \mathrm{~ns})$ was utilized. Measurements of the modulation of the sensing dye in the presence of the reference film were also performed.

The intensity decays were recovered from the frequency-domain data in terms of a multiexponential model:

$$
I(t)=\sum_{i=1}^{n} \alpha_{i} e^{-t / \tau_{i}}
$$

where the preexponential factors $\alpha_{i}$ are the amplitude of each component, $\Sigma \alpha_{i}=1.0, \tau_{i}$ are the decay times, and $n$ are the numbers of exponential components. These values were determined by a nonlinear least squares analysis as described previously (Gratton $e t$ al., 1984; Lakowicz et al., 1984). Mean lifetimes were calculated by:

$$
\langle\tau\rangle=\frac{\sum_{i} \alpha_{i} \tau_{i}^{2}}{\sum_{i} \alpha_{i} \tau_{i}}=\sum_{i} f_{i} \tau_{i}
$$

where $f_{i}$ is the fractional steady-state contribution of each component to the total emission, and $\Sigma f_{i}$ is normalized to unity. $f_{i}$ is given by

$$
f_{i}=\frac{\alpha_{i} \tau_{i}}{\sum_{j} \alpha_{j} \tau_{j}}
$$

The best fits were obtained by minimizing the $\chi_{R}^{2}$ values:

$$
\chi_{R}^{2}=\frac{1}{v} \sum_{\omega}\left[\left(\frac{\varphi_{\omega}-\varphi_{c \omega}}{\delta \varphi}\right)^{2}+\left(\frac{m_{\omega}-m_{c \omega}}{\delta m}\right)^{2}\right]
$$

where $v$ is the number of degrees of freedom, and $\varphi_{\omega}$ and $m_{\omega}$ are the experimental phase and modulation, respectively. The subscript $c$ is used to indicate calculated values for assumed values of $\alpha_{i}$ and $\tau_{i}$, and $\delta \varphi$ and $\delta m$ are the experimental uncertainties.

Prototype device The hardware and software of the prototype device and the measurement procedures of ratiometric sensing were described previously (Kostov et al., 2000, 2001). The rightangle 
format was used for the dual-frequency ratiometric measurements, and the reference film was placed inside the cuvette. In-house lowcost excitation and detection systems were employed. The excitation source was a UV LED, directly driven from the TTL output of $200 \mathrm{MHz}$ lock-in amplifier SR844 (Stanford Research Systems, Sunnyvale, USA) and filtered using a $380 \pm 30 \mathrm{~nm}$ interference filter (Intor, Soccorro, USA). Fluorescence emission was measured through a 400LH long-pass filter (Andover, Salem, USA), using a C5460 avalanche photodiode (APD) module (Hamamatsu, Bridgewater, USA) with a cut-off frequency of 10 MHz. Additionally, a 650SP short-pass filter (Andover, Salem, USA) was used to adjust the desired ratio. The signal from the APD module was fed into the lock-in amplifier for detection. The amplifier was controlled through the serial port by a computer running in-house developed software using Labview 5.0 (National Instruments, Austin, USA). The algorithm of the operation was as follows: (a) set the frequency to $25 \mathrm{kHz}$; (b) read the output 10 times and average; (c) set the frequency to $1 \mathrm{MHz}$; (d) read the output 10 times and average; and (e) calculate the ratio of the first and second averages. All of the measurements were performed without protection from the ambient light.

\section{Results and Discussion}

The chemical structure of LysoSensor DND-192 is shown in Fig. 1. LysoSensor DND-192 exhibits a significant change in fluorescence intensity upon acidification (Fig. 2). The change is about 50 times when proceeding from $\mathrm{pH} 8.91$ to $\mathrm{pH} 5.96$. However, this is not a spectral change, but only a change in<smiles>CN(C)Cc1c2ccccc2c(CN(C)C)c2ccccc12</smiles>

Fig. 1. Chemical structure of LysoSensor DND-192.

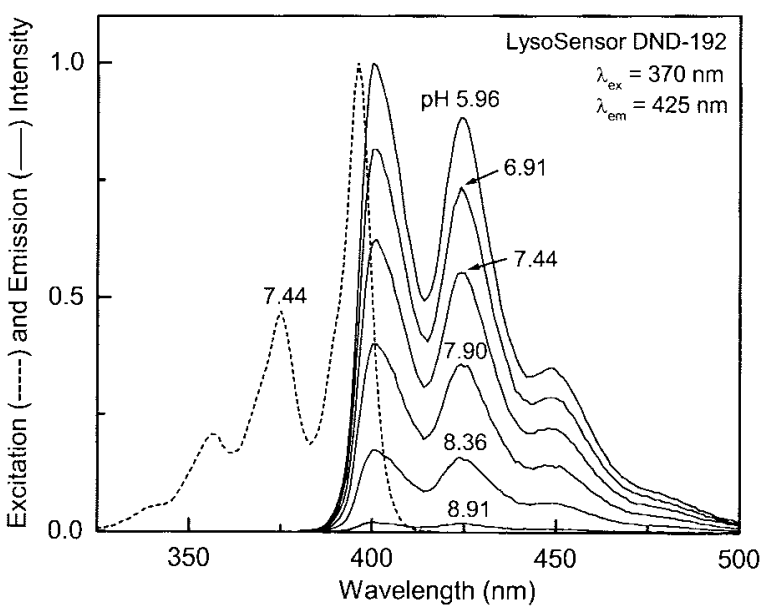

Fig. 2. Excitation (-----) and emission (-) spectra of LysoSensor DND-192 at different $\mathrm{pH}$ values. intensity. Therefore, the dye cannot be used for direct ratiometric measurements. Furthermore, the dye shows no significant change in the phase modulation characteristics (Fig. 3), which suggests that the unprotonated form of the dye is non-fluorescent. This hypothesis is strongly supported by the single exponential fit of the data (Table 1). The dye exhibits a relatively short lifetime, about 12 ns (Table 1), which makes it a perfect candidate for ratiometric measurements through lifetime discrimination.

The emission spectra of the sensing and reference dyes are presented in Fig. 4. The short- and long-dashed lines represent transmission curves of a $400 \mathrm{~nm}$ long-pass filter and a

Table 1. Multiexponential intensity decay analyses of LysoSensor DND-192 in different $\mathrm{pH}$ buffers

\begin{tabular}{cccccc}
\hline $\mathrm{pH}$ & $n^{\mathrm{a}}$ & $\begin{array}{c}\tau_{i} \\
(\mathrm{~ns})\end{array}$ & $f_{i}^{\mathrm{b}}$ & $\begin{array}{c}<\tau>^{\mathrm{b}} \\
(\mathrm{ns})\end{array}$ & $\chi_{R}^{2{ }^{\mathrm{c}}}$ \\
\hline 3.94 & 1 & 12.45 & 1.000 & 12.45 & 3.2 \\
9.16 & $2^{\mathrm{d}}$ & 11.82 & 0.994 & 11.77 & 3.9 \\
& & 2.87 & 0.006 & & \\
\hline
\end{tabular}

${ }^{\mathrm{a}}$ Number of decay times in the multiexponential fit.

${ }^{b}$ Fractional intensities $f_{i}$ and mean lifetimes $\langle\tau\rangle$ were calculated using Eqs. (7) and (6), respectively.

${ }^{c}$ The $\chi_{R}^{2}$ values were calculated by Eq. (8). The standard errors of phase angle and modulation were set at 0.2 degree and 0.005 , respectively.

${ }^{\mathrm{d}}$ The appearance of a second lifetime is probably due to scattered light.

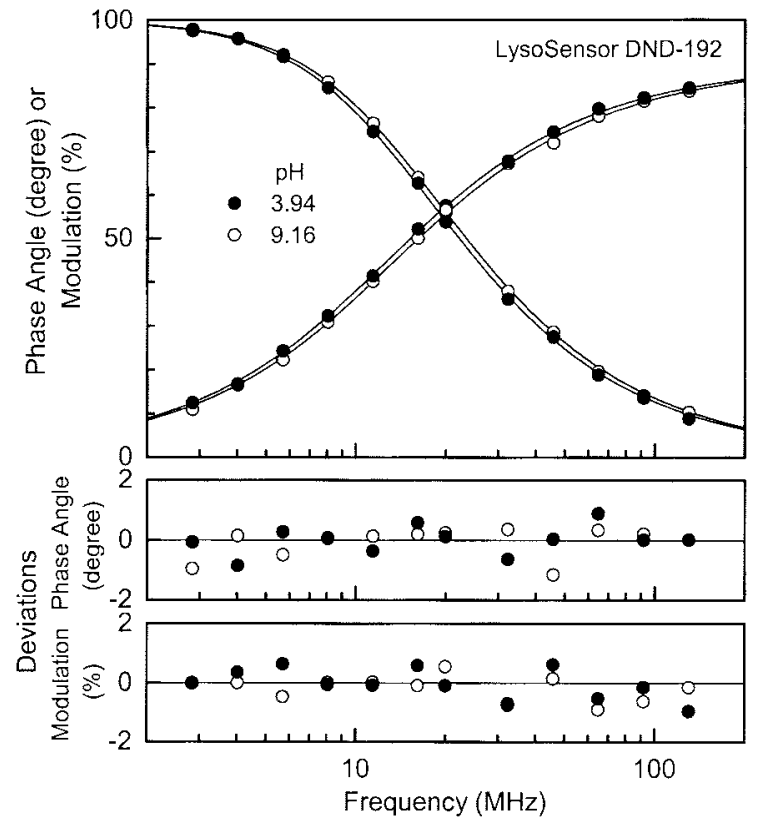

Fig. 3. Phase and modulation responses of LysoSensor DND-192 at $\mathrm{pH} 3.94$ and 9.16. The lines in the upper panel show the best fits to the experimental data. The middle and lower panels show the plots of residuals between the experimental data and fitted curves. 


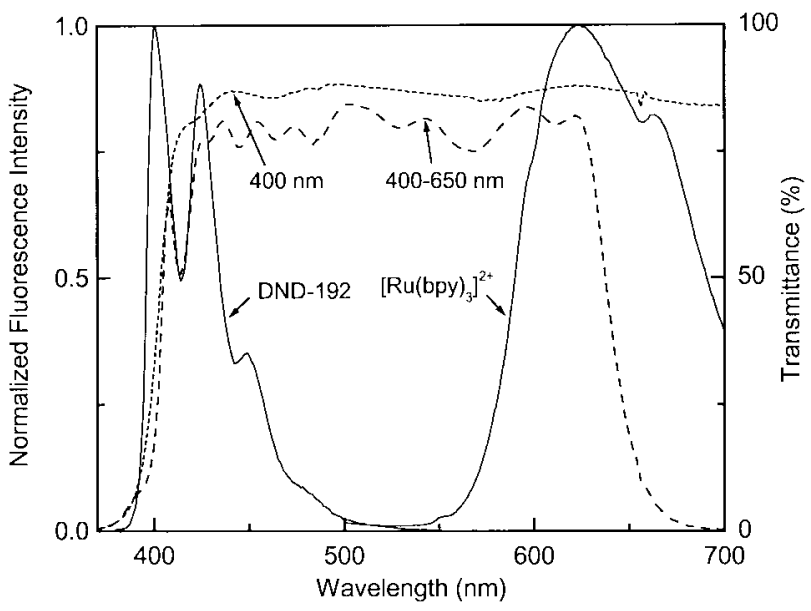

Fig. 4. Emission spectra of LysoSensor DND-192 and $\left[\mathrm{Ru}(\mathrm{bpy})_{3}\right]^{2+}$. Also shown are transmission curves of a $400 \mathrm{~nm}$ long-pass filter and a combination of a $400 \mathrm{~nm}$ long-pass filter and a $650 \mathrm{~nm}$ short-pass filter.

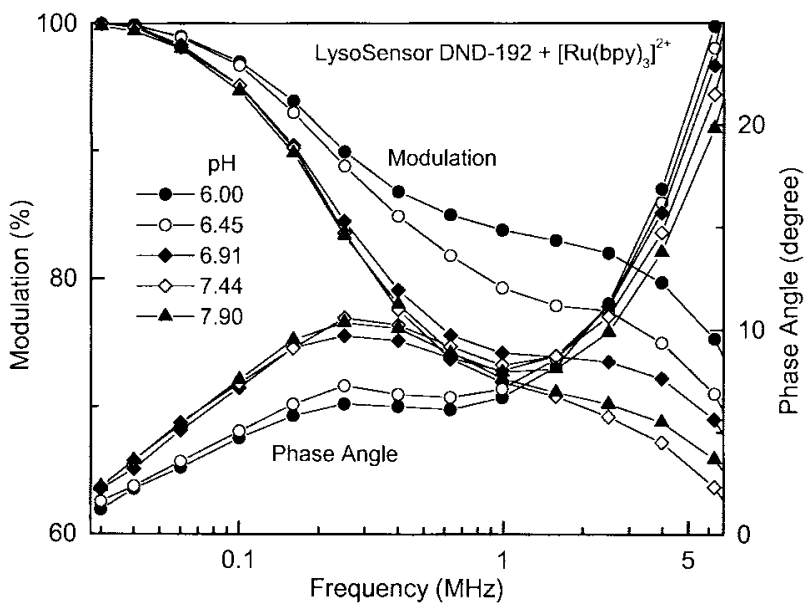

Fig. 5. Phase and modulation responses of LysoSensor DND-192 at different $\mathrm{pH}$ values in the presence of the $\left[\mathrm{Ru}(\mathrm{bpy})_{3}\right]^{2+}$ reference film.

combination of a $400 \mathrm{~nm}$ long-pass filter and a $650 \mathrm{~nm}$ shortpass filter, respectively. Using filters with different cut-off wavelengths allows the contributions from different emissions to vary, and consequently the actual value of the ratio.

The present methodology relies on the use of a semiconductor light source for excitation. LEDs are easily modulated up to hundreds of MHz (Sipior et al., 1996). Their agility is crucial for the method. However, the actual modulation frequencies depend on the lifetime of the reference. As the lifetime of $\left[\mathrm{Ru}(\mathrm{bpy})_{3}\right]^{2+}$ in cellulose acetate film is 927 ns (Kostov, 2001), we chose the frequencies of 25 $\mathrm{kHz}$ and $1 \mathrm{MHz}$ in which the requirements of Eqs. (2) and (3) are fulfilled. At the lower frequency, nearly $100 \%$ modulation of both emissions was achieved, while at $1 \mathrm{MHz}$ only the emission from the LysoSensor DND-192 is modulated. This is also confirmed by the modulation measurements on the

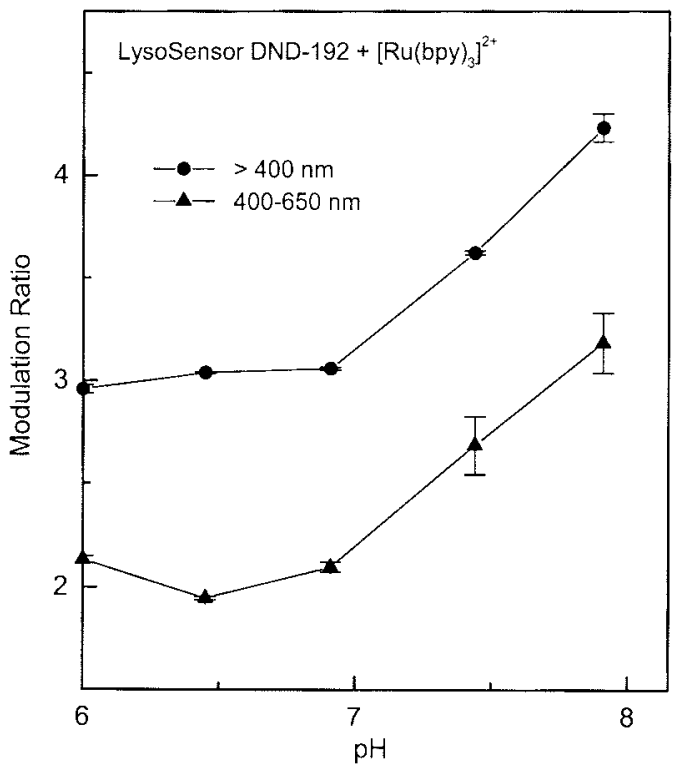

Fig. 6. The $\mathrm{pH}$ calibration curves for LysoSensor DND-192 in the presence of the $\left[R u(b p y)_{3}\right]^{2+}$ reference film using different filters. Each point represents the mean \pm SEM of 3 determinations.

frequency-domain fluorimeter (Fig. 5). As expected, there is a plateau in the modulation graphs for frequencies between 0.63 and $2.5 \mathrm{MHz}$. Any frequency in this range could be selected. As the method does not depend on frequency stability, very low-cost electronics could be used.

The emission spectra of most fluorophores usually extend far in red. The spectral overlap decreases the sensitivity of all of the wavelength-ratiometric measurements. In the present method, the emissions are selected through the modulation frequencies of the excitation light (rather than intensity measurements at different wavelengths). This greatly enhances the accuracy of the measurements. Even a complete overlap of the emission spectra poses no problem. A simple long-pass filter for the exclusion of only the excitation light could be used, while the full emission spectra are observed and collected. This strongly increases the output signal level and allows the use of less sensitive photodetectors. As a result, the whole system (both excitation and emission units) is entirely a semiconductor. It performs well, even in the presence of strong ambient light, without destroying the detector.

Actual $\mathrm{pH}$ calibration results using LysoSensor DND-192 are shown in Fig. 6. As can be seen, the ratiometric $\mathrm{pH}$ sensing that uses LysoSensor DND-192 resulted in easy and reliable $\mathrm{pH}$ measurements within the physiological range. The desired range of the ratio values can be easily adjusted using different combinations of filters in the excitation and/or emission path(s).

The use of LysoSensor DND-192 with the new ratiometric $\mathrm{pH}$ measurements can be applied to a whole family of LysoSensors. The simplicity of the excitation and detection 
system, the need for only long- and/or short-pass filter(s), enhanced levels of the output signal, and cost efficiency of this system can result in useful applications in a variety of fields.

\section{References}

Abugo, O. O., Nair, R. and Lakowicz, J. R. (2000) Fluorescence properties of rhodamine 800 in whole blood and plasma. Anal. Biochem. 279, 142-150.

Bae, J.-S. and Lee, S.-T. (2001) The human PTK6 interacts with a $23-\mathrm{kDa}$ tyrosine-phosphorylated protein and is localized in cytoplasm in breast carcinoma T-47D cells. J. Biochem. Mol. Biol. 34, 33-38.

Gratton, E., Lakowicz, J. R., Maliwal, B. P., Cherek, H. and Laczko, G. (1984) Resolution of mixtures of fluorophores using variable-frequency phase and modulation data. Biophys. J. 46, 478-486.

Kostov, Y., Harms, P., Pilato, R. S. and Rao, G. (2000) Ratiometric oxygen sensing: detection of dual-emission ratio through a single emission filter. Analyst 125, 1175-1178.

Kostov, Y., Harms, P., and Rao, G. (2001) Ratiometric sensing using dual-frequency lifetime discrimination. Anal. Biochem. 297, 105-108.

Laczko, G., Gryczynski, I., Gryczynski, Z., Wiczk, W., Malak, H. and Lakowicz, J. R. (1990) $10 \mathrm{GHz}$ fluorimeter. Rev. Sci. Instr. 61, 2331-2337.

Lakowicz, J. R. (1999) Principles of Fluorescence spectroscopy, 2nd ed., pp. 25-61, Kluwer Academic/Plenum Publishers, New York.

Lakowicz, J. R., Castellano, F. N., Dattelbaum, J. D., Tolosa, L., Rao, G. and Gryczynski, I. (1998) Low-frequency modulation sensors using nanosecond fluorophores. Anal. Chem. 70, 51155121.

Lakowicz, J. R., Gratton, E., Laczko, G., Cherek, H. and Limkeman, M. (1984) Analysis of fluorescence decay kinetics from variable-frequency phase shift and modulation data. Biophys. J. 46, 463-477.

Lakowicz, J. R., Laczko, G. and Gryczynski, I. (1986) $2 \mathrm{GHz}$ fluorimeter. Rev. Sci. Instr. 57, 2499-2506.

Lee, J. -C. and Lim K. -T. (2001) Inhibitory effects of the ethanol extract of Ulmus davidiana on apoptosis induced by glucoseglucose oxidase and cytokine production in cultured mouse primary immune cells. J. Biochem. Mol. Biol. 34, 463-471.

Lin, H. -J., Herman, P., Kang, J. S. and Lakowicz, J. R. (2001) Fluorescence lifetime characterization of novel low-pH probes. Anal. Biochem. 294, 118-125.

Nedergaard, M., Desai, S. and Pulsinelli, W. (1990) Dicarboxydichlorofluorescein: a new fluorescent probe for measuring acidic intracellular pH. Anal. Biochem. 187, 109-114.

Sipior, J., Carter, J. M., Lakowicz, J. R. and Rao, G. (1996) Single quantum well light-emitting diodes demonstrated as excitation sources for nanosecond phase modulation fluorescence lifetime measurements. Rev. Sci. Instr. 67, 37953798 .

Slavik, J., Lanz, E. and Cimprich, P. (1999) Measurement of individual intracellular $\mathrm{pH}$ and membrane potential values in living cells; in Proceedings of SPIE, Bornhop, D. J., Contag, C. H. and Sevick-Muraca, E. M. (eds), pp. 76-83, SPIE-The International Society for Optical Engineering, Bellingham, Washington.

Uh, H. S., Choi, J. H., Byun, S. M., Kim, S. Y. and Lee, H. H. (2001) Cloning, sequencing and baculovirus-based expression of fusion-glycoprotein D gene of Herpes simplex virus type 1 (F). J. Biochem. Mol. Biol. 34, 371-378.

Whitaker, J. E., Haugland, R. P. and Prendergast, F. G. (1991) Spectral and photophysical studies of benzo[c]xanthene dyes: dual emission pH sensors. Anal. Biochem. 194, 330-344.

Zignani, M., Drummond, D. C., Meyer, O., Hong, K. and Leroux, J. C. (2000) In vitro characterization of a novel polymericbased $\mathrm{pH}$-sensitive liposome system. Biochim. Biophys. Acta 1463, 383-394. 